\title{
Braid group action on the module category of quantum affine algebras
}

\author{
By Masaki KashiwarA, M.J.A., ${ }^{* 1), * 2), * 3)}$ Myungho KIM, ${ }^{* 4)}$ Se-jin $\mathrm{OH}^{* 5)}$ and Euiyong PARK ${ }^{* 6)}$ \\ (Contributed by Masaki KashiwarA, M.J.A., Feb. 12, 2021)
}

\begin{abstract}
Let $\mathfrak{g}_{0}$ be a simple Lie algebra of type $\mathrm{ADE}$ and let $U_{q}^{\prime}(\mathfrak{g})$ be the corresponding untwisted quantum affine algebra. We show that there exists an action of the braid group $B\left(\mathfrak{g}_{0}\right)$ on the quantum Grothendieck ring $\mathcal{K}_{t}(\mathfrak{g})$ of Hernandez-Leclerc's category $\mathscr{C}_{\mathfrak{g}}^{0}$. Focused on the case of type $A_{N-1}$, we construct a family of monoidal autofunctors $\left\{\mathscr{S}_{i}\right\}_{i \in \mathbf{Z}}$ on a localization $\mathcal{T}_{N}$ of the category of finite-dimensional graded modules over the quiver Hecke algebra of type $A_{\infty}$. Under an isomorphism between the Grothendieck ring $K\left(\mathcal{T}_{N}\right)$ of $\mathcal{T}_{N}$ and the quantum Grothendieck ring $\mathcal{K}_{t}\left(A_{N-1}^{(1)}\right)$, the functors $\left\{\mathscr{S}_{i}\right\}_{1 \leq i \leq N-1}$ recover the action of the braid group $B\left(A_{N-1}\right)$. We investigate further properties of these functors.
\end{abstract}

Key words: Quantum affine algebra; quantum Grothendieck ring; braid group action; quiver Hecke algebra; R-matrix.

1. Introduction. The monoidal category $\mathscr{C}_{\mathfrak{g}}$ of finite-dimensional representations of a quantum affine algebra $U_{q}^{\prime}(\mathfrak{g})$ has been extensively investigated because of its rich structure. Among various approaches, Nakajima [14], VaragnoloVasserot [16], and Hernandez [3] studied $t$-deformations of the Grothendieck ring of $\mathscr{C}_{\mathfrak{g}}$. These $t$-deformations are interesting, because they provide a way to calculate the $q$-character of simple representations. There is a full subcategory $\mathscr{C}_{\mathfrak{g}}^{0}$ of $\mathscr{C}_{\mathfrak{g}}$, introduced by Hernandez and Leclerc in [4], which contains an essential information on $\mathscr{C}_{\mathfrak{g}}$ but has a smaller set of the classes of simple modules. The Grothendieck ring of $\mathscr{C}_{\mathfrak{g}}^{0}$ is isomorphic to the polynomial ring in countably many variables, while that of $\mathscr{C}_{\mathfrak{g}}$ is the one in uncountably many variables. For the cases where $\mathfrak{g}$ is one of un-

2010 Mathematics Subject Classification. Primary 17B37, 20F36; Secondary 18D10.

*1) Kyoto University Institute for Advanced Study, Yoshida Ushinomiya-cho, Sakyo-ku, Kyoto 606-8501, Japan.

*2) Research Institute for Mathematical Sciences, Kyoto University, Kitashirakawa-Oiwakecho, Sakyo-ku, Kyoto 6068502, Japan.

*3) Korea Institute for Advanced Study, Cheongryangridong, Dongdaemun-gu, 207-43, Seoul 02455, Korea.

*4) Department of Mathematics, Kyung Hee University, 26 Kyunghee-daero, Dongdaemun-gu, Seoul 02447, Republic of Korea.

*5) Department of Mathematics, Ewha Womans University, 52, Ewhayeodae-gil, Seodaemun-gu, Seoul 03760, Republic of Korea.

*6) Department of Mathematics, University of Seoul, 163 Seoulsiripdaero, Dongdaemun-gu, Seoul 02504, Republic of Korea. twisted ADE types, a $t$-deformation $\mathcal{K}_{t}(\mathfrak{g})$ of the Grothendieck ring of $\mathscr{C}_{\mathfrak{g}}^{0}$, called the quantum Grothendieck ring, was investigated from a ring theoretic point of view in [5]. It turns out that the $\mathbf{C}\left(t^{1 / 2}\right)$-algebra $\mathcal{K}_{t}(\mathfrak{g})$ has an interesting presentation: there is a set of generators consisting of a countable infinite number of copies of DrinfeldJimbo generators of a half of the quantum group $U_{t}\left(\mathfrak{g}_{0}\right)$, and they satisfy the quantum Serre relations in a copy, $t$-boson relations between adjacent copies, and $t$-commutation relations between nonadjacent copies. This presentation reflects the following feature of the category $\mathscr{C}_{\mathfrak{g}}^{0}$ : for each choice of a Dynkin quiver $Q$ with an additional data, they defined a monoidal subcategory $\mathscr{C}_{Q}$ of $\mathscr{C}_{\mathfrak{g}}^{0}$ such that the quantum Grothendieck ring of $\mathscr{C}_{Q}$ is isomorphic to the half $U_{t}^{-}\left(\mathfrak{g}_{0}\right)$ of the quantum group $U_{t}\left(\mathfrak{g}_{0}\right)$, and all the fundamental representations in $\mathscr{C}_{\mathfrak{g}}^{0}$ can be obtained from those in $\mathscr{C}_{Q}$ by taking functors $\mathscr{D}^{m}(m \in \mathbf{Z})$. Here $\mathscr{D}$ is the contravariant functor taking the right dual.

One of main results of this paper is that there exists an action of the braid group $B\left(\mathfrak{g}_{0}\right)$ of type $\mathfrak{g}_{0}$ on the quantum Grothendieck ring $\mathcal{K}_{t}(\mathfrak{g})$ (Theorem 2.3 ). Since we give the action explicitly, the braid relations can be checked by the presentation of $\mathcal{K}_{t}(\mathfrak{g})$. Recall that the blocks of the category $\mathscr{C}_{\mathfrak{g}}^{0}$ is parameterized by the root lattice of $\mathfrak{g}_{0}$ and the tensor product is compatible with the addition on the root lattice [10]. It turns out that the action of the generators $\sigma_{i}$ of $B\left(\mathfrak{g}_{0}\right)$ on $\mathcal{K}_{t}(\mathfrak{g})$ correspond to the reflections with respect to the simple roots $\alpha_{i}$ on the 
root lattice. Indeed, the action of $\sigma_{i}$ is related with Saito's reflection functor as seen in Theorem 2.4.

We conjecture that the braid group action can be lifted to the action on the monoidal category $\mathscr{C}_{\mathrm{g}}^{0}$. We show that it is the case when $\mathfrak{g}$ is of type $A_{N-1}^{(1)}$. A key point of view is the use of a rigid monoidal category $\mathcal{T}_{N}$ which is constructed out of the category $\mathcal{A}$ of finite-dimensional graded modules over the quiver Hecke algebra $R^{A_{\infty}}$ of type $A_{\infty}$ [7]. It is a certain localization of $\mathcal{A}$ and there is a monoidal functor $\mathcal{F}_{N}$ from $\mathcal{T}_{N}$ to $\mathscr{C}_{A_{N-1}^{(1)}}^{0}$ which sends simple objects to simple objects. Moreover this functor induces an isomorphism between the Grothendieck ring $K\left(\mathcal{T}_{N}\right)$ and the quantum Grothendieck ring $\mathcal{K}_{t}\left(A_{N-1}^{(1)}\right)$. It is summarized by the diagram

$$
K\left(\mathcal{T}_{N}\right) \stackrel{\sim}{\rightarrow} \mathcal{K}_{t}\left(A_{N-1}^{(1)}\right) \stackrel{t=1}{\longrightarrow} K\left(\mathscr{C}_{A_{N-1}^{(1)}}^{0}\right) .
$$

Hence the category $\mathcal{T}_{N}$ can be understood as a graded lift of $\mathscr{C}_{A_{N-1}^{(1)}}^{0}$ as a rigid monoidal category.

We show that there is a family of monoidal autofunctors $\left\{\mathscr{S}_{i}\right\}_{1 \leq i \leq N-1}$ on the category $\mathcal{T}_{N}$ which recover the action of the braid group $B\left(A_{N-1}\right)$ under the isomorphism between $K\left(\mathcal{T}_{N}\right)$ and $\mathcal{K}_{t}\left(A_{N-1}^{(1)}\right)$ (Theorem 3.1, Theorem 3.3). There is a general procedure, developed in [11], to construct monoidal functors between the categories of modules over quiver Hecke algebras, and a similar procedure can be applied for the category $\mathcal{T}_{N}$. This is a main advantage in working on the category $\mathcal{T}_{N}$ rather than the category $\mathscr{C}_{A^{(1)}}^{0}$.

Finally we provide several consequences of the existence of such functors $\mathscr{S}_{i}$. For a simple object $L$ which belongs to an orbit of $L(i)$ for some $i$ under the action $B\left(A_{N-1}\right)$, one can define an automorphism $s_{L}$ which has similar properties with the automorphisms $s_{i}$ (Theorem 4.2). Moreover $s_{L(i)}$ coincides with $s_{i}$.

This paper is an announcement whose details will appear elsewhere.

Convention 1.1. Throughout this paper, we keep the following conventions.

(a) For a statement $\mathrm{P}, \delta(\mathrm{P})$ is 1 or 0 according that $P$ is true or not.

(b) For $k, \ell \in \mathbf{Z}$ and $s \in \mathbf{Z}_{\geq 1}$, we write $k \equiv_{s} \ell$ if $s$ divides $k-\ell$ and $k \neq_{s} \ell$, otherwise.

2. Braid group action on the quantum Grothendieck rings. Let $\mathfrak{g}_{0}$ be a finite-dimensional simple Lie algebra of simply-laced type with a Cartan matrix $\mathbf{A}=\left(a_{i j}\right)_{i, j \in I_{0}}, \mathfrak{g}$ the untwisted affine Kac-Moody algebra associated with $\mathfrak{g}_{0}$, and $U_{q}^{\prime}(\mathfrak{g})$ the quantum affine algebra associated with $\mathfrak{g}$. We take the algebraic closure of $\mathbf{C}(q)$ inside $\bigcup_{m>0} \mathbf{C}\left(\left(q^{1 / m}\right)\right)$ as the base field $\mathbf{k}$ for $U_{q}^{\prime}(\mathfrak{g})$. Let $\mathscr{C}_{\mathfrak{g}}$ be the category of finite-dimensional integrable modules over $U_{q}^{\prime}(\mathfrak{g})$. There is a family $\left\{V\left(\varpi_{i}\right)_{c} \mid i \in\right.$ $\left.I_{0}, c \in \mathbf{k}^{\times}\right\}$in $\mathscr{C}_{\mathfrak{g}}$, called the fundamental representations.

Following [4], we denote by $\mathscr{C}_{\mathfrak{g}}^{0}$ the smallest full subcategory of the category $\mathscr{C}_{\mathfrak{g}}$ which is stable under taking subquotients, extensions, tensor products and contains

$$
\left\{V\left(\varpi_{i}\right)_{(-q)^{p}} \mid i \in I_{0}, p \equiv d(1, i) \bmod 2\right\},
$$

where $d(i, j)$ is the distance between the vertices $i$ and $j$ in the Dynkin diagram of $\mathfrak{g}_{0}$. Here $1 \in I_{0}$ is an arbitrarily chosen element. Then the complexified Grothendieck ring $\mathbf{C} \otimes_{\mathbf{z}} K\left(\mathscr{C}_{\mathfrak{g}}^{0}\right)$ of $\mathscr{C}_{\mathfrak{g}}^{0}$ has a $t$-deformation $\mathcal{K}_{t}(\mathfrak{g})$, called the quantum Grothendieck ring of $\mathscr{C}_{\mathfrak{g}}^{0}$. To each simple module $S$ in $\mathscr{C}_{\mathfrak{g}}^{0}$, we can associate an element $[S]_{t}$ of $\mathcal{K}_{t}(\mathfrak{g})$ and we have $\mathcal{K}_{t}(\mathfrak{g})=\bigoplus \mathbf{C}\left(t^{1 / 2}\right)[S]_{t}$. Here $S$ ranges over the set of the isomorphism classes of simple modules in $\mathscr{C}_{\mathrm{g}}^{0}$.

Let $Q$ be a Dynkin quiver with type $\mathfrak{g}_{0}$, and let $\phi_{Q}$ be a height function, i.e., it associates an integer $\phi_{Q}(i)$ to each vertex $i$ of $Q$ such that $\phi_{Q}(i)=$ $\phi_{Q}(j)+1$ if $i \rightarrow j$. We assume further that $\phi_{Q}(1) \in 2 \mathbf{Z}$. A pair $Q=\left(Q, \phi_{Q}\right)$ is called a Q-data.

For a sink $i$ of $Q$, let $s_{i} Q:=\left(s_{i} Q, \phi_{s_{i} Q}\right)$ be the Q-data consisting of the Dynkin quiver $s_{i} Q$ obtained from $Q$ by reversing the arrows of $Q$ adjacent to $i$ and the height function $\phi_{s_{i} Q}$ of $s_{i} Q$ given by $\phi_{s_{i} Q}(j)=\phi_{Q}(j)+2 \delta_{i, j}$.

To a Q-data $Q$, Hernandez-Leclerc [5] associated a full monoidal subcategory $\mathscr{C}_{Q}$ of $\mathscr{C}_{\mathfrak{g}}^{0}$, and a monoidal functor $\mathcal{F}_{Q}: R_{\mathfrak{g}_{0}}-\bmod \rightarrow \mathscr{C}_{Q}$ is constructed in $[2,6]$, and Fujita $[1,2]$ proved that $\mathcal{F}_{Q}$ is an equivalence of categories. Here, $R_{\mathfrak{g}_{0}}$-mod is the monoidal category of finite-dimensional modules (with nilpotent actions of the generators $x_{k}$ ) over the quiver Hecke algebra $R_{\mathfrak{g}_{0}}$ associated with $\mathfrak{g}_{0}$. Note that $\mathcal{F}_{Q}(L(i))$ is a fundamental module for any $i \in I_{0}$, where $L(i) \in R_{\mathfrak{g}_{0}}$-mod is the simple module associated with $i$.

Then, for a Q-data $Q$, we have an embedding of $\mathbf{Z}\left[t^{ \pm 1}\right]$-algebras

$$
j_{Q}: K\left(R_{\mathfrak{g}_{0}} \text {-gmod }\right) \hookrightarrow \mathcal{K}_{t}(\mathfrak{g})
$$

induced by $\mathcal{F}_{Q}$.

Let $\mathbf{K}_{t}\left(\mathfrak{g}_{0}\right)$ be the $\mathbf{C}\left(t^{1 / 2}\right)$-algebra generated by 
$\left\{y_{i, m} \mid i \in I_{0}, m \in \mathbf{Z}\right\}$ with the defining relations: For $m \in \mathbf{Z}$ and $i, j \in I_{0}$,

(a) $y_{i, m} y_{j, m}=y_{j, m} y_{i, m}$ if $a_{i j}=0$,

$$
\begin{gathered}
y_{i, m}^{2} y_{j, m}-\left(t+t^{-1}\right) y_{i, m} y_{j, m} y_{i, m}+y_{j, m} y_{i, m}^{2}=0 \\
\text { if } a_{i j}=-1,
\end{gathered}
$$

(b) $y_{i, m} y_{j, m+1}=t^{a_{i j}} y_{j, m+1} y_{i, m}+\delta_{i j}\left(1-t^{2}\right)$,

(c) $y_{i, m} y_{j, p}=t^{(-1)^{p-m+1} a_{i j}} y_{j, p} y_{i, m}$, for $p>m+1$.

Remark 2.1. We change $t$ into $t^{-1}$ in the presentation in [5].

Theorem 2.2 ([5], Theorem 7.3). Let $Q$ be a $Q$-data. Then there is an isomorphism $\iota_{Q}: \mathbf{K}_{t}\left(\mathfrak{g}_{0}\right) \stackrel{\sim}{\rightarrow}$ $\mathcal{K}_{t}(\mathfrak{g})$ such that $\iota_{Q}\left(y_{i, m}\right)$ is equal to $\left[\mathscr{D}^{m} \mathcal{F}_{Q}(L(i))\right]_{t}$, where $L(i)$ is the simple module in $R_{\mathfrak{g}_{0}}$-mod corresponding to $i \in I_{0}$.

Let $B\left(\mathfrak{g}_{0}\right)$ be the Braid group associated with $\mathfrak{g}_{0}$. It is generated by $\left\{\sigma_{i} \mid i \in I_{0}\right\}$ with the defining relations

$$
\begin{aligned}
\sigma_{i} \sigma_{j} \sigma_{i} & =\sigma_{j} \sigma_{i} \sigma_{j} \quad \text { if } a_{i j}=-1, \\
\sigma_{i} \sigma_{j} & =\sigma_{j} \sigma_{i} \quad \text { if } a_{i j}=0 .
\end{aligned}
$$

One of our main theorems is the following

Theorem 2.3. The Braid group $B\left(\mathfrak{g}_{0}\right)$ acts on $\mathbf{K}_{t}\left(\mathfrak{g}_{0}\right)$ by the following formulas:

$$
\begin{array}{r}
\sigma_{i}\left(y_{j, m}\right) \\
= \begin{cases}y_{j, m+\delta_{i j}} & \text { if } a_{i j} \neq-1, \\
\frac{t^{1 / 2} y_{j, m} y_{i, m}-t^{-1 / 2} y_{i, m} y_{j, m}}{t-t^{-1}} & \text { if } a_{i j}=-1,\end{cases} \\
\sigma_{i}^{-1}\left(y_{j, m}\right) \\
= \begin{cases}y_{j, m-\delta_{i j}} & \text { if } a_{i j} \neq-1, \\
\frac{t^{1 / 2} y_{i, m} y_{j, m}-t^{-1 / 2} y_{j, m} y_{i, m}}{t-t^{-1}} & \text { if } a_{i j}=-1 .\end{cases}
\end{array}
$$

Theorem 2.4. Let $i$ be a sink of a $Q$-data $Q$. Then the following diagrams commute:
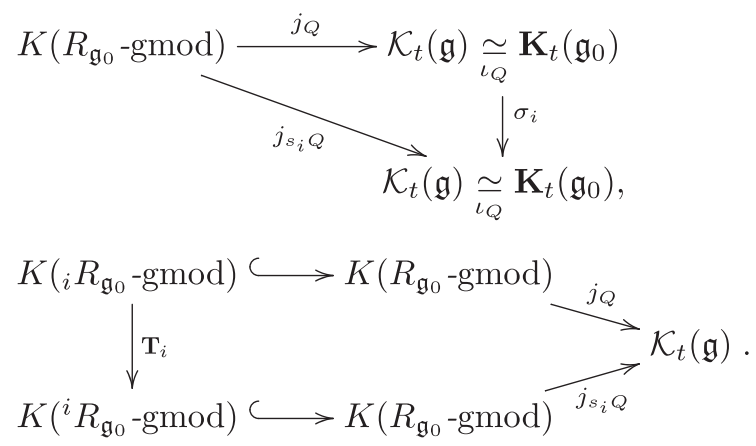

Here, ${ }_{i} R_{\mathfrak{g}_{0}}$-gmod (resp. ${ }^{i} R_{\mathfrak{g}_{0}}$-gmod) is the full subcategory of $R_{\mathfrak{g}_{0}}$-gmod consisting of graded modules $M$ with $E_{i}^{*} M=0$ (resp. $E_{i} M=0$ ), and $\mathbf{T}_{i}$ is the reflection functor due to $\mathrm{S}$. Kato $[12,13]$ (cf. Y. Saito [15]). For $E_{i}$ and $E_{i}^{*}$, see for example, [8].

3. The category $\mathcal{T}_{N}$ and reflection functors. Let $J$ be the index set of simple roots of the root system $A_{\infty}$. One can identify $J$ with $\mathbf{Z}$ and the root lattice $Q$ is the subspace of $\bigoplus_{a \in \mathbf{Z}} \mathbf{Z} \varepsilon_{a}$ generated by $\alpha_{a}=\varepsilon_{a}-\varepsilon_{a+1}$ for $a \in \mathbf{Z}$. Let $R^{A_{\infty}}$ be the symmetric quiver Hecke algebra of type $A_{\infty}$ over $\mathbf{k}$ with the choice of parameters

$$
Q_{i j}(u, v)=\delta(i \neq j)(u-v)^{\delta(j=i+1)}(v-u)^{\delta(j=i-1)}
$$

for $i, j \in J$. It is a family $\left\{R^{A_{\infty}}(\beta)\right\}_{\beta \in Q^{+}}$of associative $\mathbf{Z}$-graded $\mathbf{k}$-algebras, where $\mathbf{Q}^{+}=\sum_{i \in J} \mathbf{Z}_{\geq 0} \alpha_{i}$ is the positive root lattice of type $A_{\infty}$. Each $R^{A_{\infty}}(\beta)$ is generated by $\{e(\nu)\}_{\nu \in J^{\beta}}, \quad\left\{x_{k}\right\}_{1 \leq k \leq n}$ and $\left\{\tau_{m}\right\}_{1 \leq m \leq n-1}$, where $n=|\beta|:=\sum_{i \in I} n_{i}$ with $\beta=\sum_{i \in J} n_{i} \alpha_{i}$, and $J^{\beta}:=\left\{\nu \in J^{n} \mid \alpha_{\nu_{1}}+\cdots+\alpha_{\nu_{n}}=\right.$ $\beta$ \}. See [7] for a set of defining relations of $R^{A_{\infty}}(\beta)$. Note that there is an embedding of $R^{A_{\infty}}(\beta) \otimes$ $R^{A_{\infty}}(\gamma)$ into $R^{A_{\infty}}(\beta+\gamma)$. Hence the category $\mathcal{A}=$ $\bigoplus_{\beta \in Q^{+}} R^{A_{\infty}}(\beta)$-gmod of finite-dimensional graded $R^{A_{\infty}}$-modules is a monoidal category whose tensor product is given by the convolution product:

$$
M \circ N:=R^{A_{\infty}}(\beta+\gamma) \underset{R^{A_{\infty}}(\beta) \otimes R^{A_{\infty}}(\gamma)}{\otimes}(M \otimes N) .
$$

For $M \in R^{A_{\infty}}(\beta)$-gmod, we $\operatorname{set} \operatorname{wt}(M):=-\beta$.

For each pair of integers $a, b$ with $a \leq b$, let $[a, b]$ be the interval $\{k \in \mathbf{Z} \mid a \leq k \leq b\}$, and call it a segment. For each segment $[a, b]$, let $L(a, b)$ be the one-dimensional simple graded $R^{A_{\infty} \text {-module }}$ generated by a vector $u(a, b)$ such that $e(\nu) u(a, b)=$ $\delta(\nu=(a, \ldots, b)) u(a, b)$. We set $L(a):=L(a, a)$ for $a \in \mathbf{Z}$. For each $N \geq 2$, let $\mathcal{S}_{N}$ be the smallest subcategory of $\mathcal{A}$ which is stable under taking convolution, subquotients, extensions, and containing $\{L(a, b) \mid b-a+1>N\}$. Then the quotient category $\mathcal{A} / \mathcal{S}_{N}$ equips with a new tensor product $\star$ given by

$$
X \star Y:=t^{B(\operatorname{wt}(X), \operatorname{wt}(Y))} X \circ Y,
$$

where $B(x, y):=-\sum_{k>0}\left(S^{k} x, y\right)$ for $x, y \in \bigoplus_{a \in \mathbf{Z}} \mathbf{Z} \varepsilon_{a}$ and $S$ is an automorphism on $\bigoplus_{a \in \mathbf{Z}} \mathbf{Z} \varepsilon_{a}$ given by $S\left(\varepsilon_{a}\right):=\varepsilon_{a+N}$. The category $\mathcal{T}_{N}$ is constructed in [7] as a localization of the monoidal category $\left(\mathcal{A} / \mathcal{S}_{N}, \star\right)$. The objects of $\mathcal{T}_{N}$ is the same with the ones of $\mathcal{A} / \mathcal{S}_{N}$. The group of morphisms is given by 
$\operatorname{Hom}_{\mathcal{T}_{\mathcal{N}}}(X, Y):=\underset{\lambda, \mu}{\lim _{\vec{\lambda}}} \operatorname{Hom}_{\mathcal{A} / \mathcal{S}_{N}}\left(X \circ P^{\lambda}, Y \circ P^{\mu}\right)$,

where $P^{\nu}:=\circ_{a \in \mathbf{Z}} L(a, a+N-1)^{\circ \nu_{a}}$ for $\nu \in\left(\mathbf{Z}_{\geq 0}\right)^{\oplus J}$ and the limit runs over all the pairs $(\lambda, \mu)$ such that $\operatorname{wt}\left(X \circ P^{\lambda}\right)=\operatorname{wt}\left(Y \circ P^{\mu}\right)$. It turns out that $\mathcal{T}_{N}$ is an abelian rigid monoidal category with a tensor product $\star$. We denote the right dual (resp. left dual) of $X$ by $\mathscr{D}(X)\left(\right.$ resp. $\left.\mathscr{D}^{-1}(X)\right)$. Note that $L(a, a+$ $N-1) \simeq \mathbf{1}$ in $\mathcal{T}_{N}$ for all $a \in \mathbf{Z}$. We have a chain of functors

$$
\mathcal{A} \stackrel{\mathcal{Q}_{N}}{\longrightarrow} \mathcal{A} / \mathcal{S}_{N} \stackrel{\Upsilon_{N}}{\longrightarrow} \mathcal{T}_{N} .
$$

The composition will be denoted by $\Omega_{N}$. Note that the Grothendieck ring $K\left(\mathcal{T}_{N}\right)$ is a $\mathbf{Z}\left[t^{ \pm 1}\right]$-algebra on which $t$ acts by the grading shift.

From now on, let $\mathfrak{g}$ be the affine Kac-Moody algebra of type $A_{N-1}^{(1)}$. We regard $\mathcal{T}_{N}$ as a $\mathbf{Z}$-graded lifting of $\mathscr{C}_{\mathfrak{g}}^{0}$ as a rigid monoidal category. Indeed there exists a monoidal functor $\mathcal{F}_{N}: \mathcal{T}_{N} \rightarrow \mathscr{C}_{\mathfrak{g}}^{0}$ which sends simples to simples. It induces an isomorphism of $\mathbf{C}\left(t^{1 / 2}\right)$-algebras $\left[\mathcal{F}_{N}\right]: \mathbf{C}\left(t^{1 / 2}\right) \otimes_{\mathbf{Z}\left[t^{ \pm 1}\right]}$ $K\left(\mathcal{T}_{N}\right) \stackrel{\sim}{\rightarrow} \mathcal{K}_{t}(\mathfrak{g}) \quad([7]$, Theorem 4.33). Under the isomorphism, the generator $y_{i, m}$ corresponds to $\left[\mathscr{D}^{m} L(i)\right]$ for $i \in \mathbf{Z}, m \in \mathbf{Z}$.

For a pair $(M, N)$ of objects in a k-linear abelian monoidal category in which every object has a finite length, we denote by $M \nabla N$ the head of $M \otimes N$ and by $M \Delta N$ the socle of $M \otimes N$, respectively.

We show that there is a family of autofunctors on $\mathcal{T}_{N}$ which recover the braid group action on the quantum Grothendieck ring $\mathcal{K}_{t}(\mathfrak{g})$. For this purpose, we adjoin a formal object $t^{1 / 2} \mathbf{1}$ into $\mathcal{T}_{N}$ such that $t^{1 / 2} \mathbf{1} \star t^{1 / 2} \mathbf{1} \simeq t \mathbf{1}$. Then the grading shift by $1 / 2$ of $X$ is given by $X \rightarrow t^{1 / 2} \mathbf{1} \star X$.

Theorem 3.1. For $i \in \mathbf{Z}$, there exists a monoidal functor

$$
\mathscr{S}_{i}: \mathcal{T}_{N} \rightarrow \mathcal{T}_{N}
$$

satisfying

$$
\mathscr{S}_{i}(L(j)) \simeq \begin{cases}\mathscr{D} L(j) & \text { if } j \equiv_{N} i, \\ t^{1 / 2}(L(j \mp 1) \nabla L(j)) & \text { if } j \equiv_{N} i \pm 1, \\ L(j) & \text { otherwise. }\end{cases}
$$

The functor $\mathscr{S}_{i}$ has an inverse

$$
\mathscr{S}_{i}^{-1}: \mathcal{T}_{N} \rightarrow \mathcal{T}_{N}
$$

satisfying
$\mathscr{S}_{i}^{-1}(L(j)) \simeq \begin{cases}\mathscr{D}^{-1} L(j) & \text { if } j \equiv_{N} i \\ t^{1 / 2}(L(j) \nabla L(j \mp 1)) & \text { if } j \equiv_{N} i \pm 1 \\ L(j) & \text { otherwise. }\end{cases}$

Let us explain briefly how to construct the functors $\mathscr{S}_{i}$. For each $j \in J$, denote $\bar{M}_{j}$ the

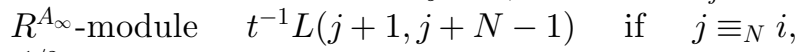
$t^{1 / 2}(L(j \mp 1) \nabla L(j))$ if $j \equiv_{N} i \pm 1$ and $L(j)$ otherwise. For each $\beta \in \mathrm{Q}^{+}$and $\mu=\left(\mu_{1}, \ldots, \mu_{m}\right) \in J^{\beta}$, set

$$
\Delta(\mu)=M_{\mu_{1}} \circ \cdots \circ M_{\mu_{m}} \text {, and } \Delta(\beta)=\bigoplus_{\mu \in J^{\beta}} \Delta(\mu),
$$

where $M_{j}$ is the affinization of $\bar{M}_{j}$. Then along a similar line with ([11], Section 4), one can show that there exists a ring homomorphism

$$
\left(R^{A_{\infty}}(\beta)\right)^{\text {opp }} \rightarrow \operatorname{End}_{\mathcal{A}^{\text {big }} / \mathcal{S}_{N}^{\text {big }}}\left(\mathcal{Q}_{N}(\Delta(\beta))\right),
$$

where $\mathcal{A}^{\text {big }} / \mathcal{S}_{N}^{\text {big }}$ is a quotient category of the category of graded $R^{A_{\infty}}$-modules which is defined in a similar way with $\mathcal{A} / \mathcal{S}_{N}$ (see [7], Section 4.4). Let $\mathcal{R}_{\beta}^{\prime}: R^{A_{\infty}}(\beta)-\operatorname{gmod} \rightarrow \mathcal{A} / \mathcal{S}_{N}$ be the restriction of a left adjoint of the functor


idal functor $\mathcal{R}: \mathcal{A} \rightarrow \mathcal{T}_{N}$, the composition

$$
\mathcal{A} \stackrel{\bigoplus_{\beta \in Q^{+}} \mathcal{R}_{\beta}^{\prime}}{\longrightarrow} \mathcal{A} / \mathcal{S}_{N} \stackrel{\Upsilon_{N}}{\longrightarrow} \mathcal{T}_{N} .
$$

Note that the family $\left\{\bar{M}_{j}\right\}_{j \in J}$ of objects in $\mathcal{T}_{N}$ satisfies for any $a \in J$ that (1) $\bar{M}_{a} \star \bar{M}_{a+1} \star \cdots \star$ $\bar{M}_{a+N-1} \simeq 1, \quad(2) \quad \operatorname{hd}\left(\bar{M}_{a} \star \bar{M}_{a+1} \star \cdots \star \bar{M}_{a+k-1}\right) \star$ $\bar{M}_{a+k}$ is not simple for $1 \leq k \leq N-1$, and (3) $\mathscr{D}^{2}\left(\bar{M}_{a}\right) \simeq \bar{M}_{a+N}$. A similar argument as the one in ([9], Section 6.1) shows that there is a monoidal functor $\mathscr{S}_{i}: \mathcal{T}_{N} \rightarrow \mathcal{T}_{N}$ such that $\mathcal{R} \simeq \mathscr{S}_{i} \circ \Omega_{N}$.

Recall that there is an automorphism $\mathrm{T}: \mathcal{T}_{N} \rightarrow$ $\mathcal{T}_{N}$ given by $L(j) \mapsto L(j+1)$ for all $j \in \mathbf{Z}$. It satisfies that $\mathrm{T}^{N} \simeq \mathscr{D}^{2}$. The functors $\left\{\mathscr{S}_{i} \mid i \in \mathbf{Z}\right\}$ satisfy the following properties.

Proposition 3.2. We have

(i) $\mathscr{S}_{i+1} \simeq \mathrm{T} \circ \mathscr{S}_{i} \circ \mathrm{T}^{-1}$ for $i \in \mathbf{Z}$,

(ii) $\mathscr{S}_{i} \circ \mathscr{D} \simeq \mathscr{D} \circ \mathscr{S}_{i}$ for $i \in \mathbf{Z}$,

(iii) $\mathscr{S}_{i} \simeq \mathscr{S}_{N+i}$ for $i \in \mathbf{Z}$,

(iv) $\mathscr{S}_{1} \mathscr{S}_{2} \cdots \mathscr{S}_{N-1} \simeq \mathrm{T}$,

(v) $\mathscr{S}_{i} \circ \mathscr{S}_{j} \simeq \mathscr{S}_{j} \circ \mathscr{S}_{i}$ for $|i-j|>2$,

(vi) $\mathscr{S}_{i} \circ \mathscr{S}_{i+1} \circ \mathscr{S}_{i} \simeq \mathscr{S}_{i+1} \circ \mathscr{S}_{i} \circ \mathscr{S}_{i+1}$ for $i \in \mathbf{Z}$.

The family of functors $\left\{\mathscr{S}_{i}\right\}_{1<i<N-1}$ recovers the braid group action in Theorem 2.3 in the case of type $A_{N-1}$.

Theorem 3.3. For each $1 \leq i \leq N-1$ the $\mathbf{Z}\left[t^{ \pm 1 / 2}\right]$-algebra automorphism on $K\left(\overline{\mathcal{T}}_{N}\right)$ induced 
by $\mathscr{S}_{i}$ is equal to $\sigma_{i}$ in Theorem 2.3 under the isomorphism $\left[\mathcal{F}_{N}\right]: \mathbf{C}\left(t^{1 / 2}\right) \otimes_{\mathbf{Z}\left[t^{ \pm 1}\right]} K\left(\mathcal{T}_{N}\right) \stackrel{\sim}{\rightarrow} \mathcal{K}_{t}(\mathfrak{g})$.

4. Reflections by root modules. Recall that for each pair of non-zero modules $(X, Y)$ of $\mathcal{A}$, there exists a distinguished nonzero morphism $\mathbf{r}_{X, Y}: t^{\Lambda(X, Y)} X \circ Y \rightarrow Y \circ X$ called the r-matrix [7]. Here, $t$ is the grading shift functor. We have $\Omega_{N}\left(\mathbf{r}_{X, Y}\right): t^{\Lambda_{N}(X, Y)} X \star Y \rightarrow Y \star X$ in $\mathcal{T}_{N}$, where $\Lambda_{N}(X, Y)=\Lambda(X, Y)-B(\operatorname{wt}(X), \operatorname{wt}(Y))+$ $B(\operatorname{wt}(Y), \operatorname{wt}(X))$.

For a pair $(X, Y)$ of objects in $\mathcal{T}_{N}$, set

$$
\mathfrak{d}(X, Y):=\frac{1}{2}\left(\Lambda_{N}(X, Y)+\Lambda_{N}(Y, X)\right) .
$$

Note that $\mathfrak{d}(X, Y)=\frac{1}{2}(\Lambda(X, Y)+\Lambda(Y, X)) \quad$ if $\Omega\left(\mathbf{r}_{X, Y}\right) \neq 0$.

A simple object $X$ in an abelian monoidal category is called real if $X \otimes X$ is simple. A real simple object $L$ in $\mathcal{T}_{N}$ is called a root module if

$$
\mathfrak{d}\left(L, \mathscr{D}^{k}(L)\right)=\delta(k= \pm 1) .
$$

For example, the objects $L(a, b)$ with $b-a+1<$ $N$ are root modules. If $L$ is a root module, then $\mathscr{D}(L), \mathscr{D}^{-1}(L)$ and $\mathscr{S}_{i}(L)$ for $i \in \mathbf{Z}$ are root modules.

The following is the main theorem of this section.

Theorem 4.1. Let $X$ be a simple object in $\mathcal{T}_{N}$. For $i \in \mathbf{Z}$, if

$$
\mathfrak{d}\left(\mathscr{D}^{k}(L(i)), X\right)=n \delta(k=a)
$$

for some $n \geq 0$ and $a \in \mathbf{Z}$, then

$$
\mathscr{S}_{i}(X) \simeq\left(\mathscr{D}^{a} L(i)\right)^{\circ n} \nabla X
$$

up to a multiple of a power of $t$.

The following is one of the applications of Theorem 4.1.

Theorem 4.2. Let $[L]$ belongs to the orbit of $L(i)$ for some $1 \leq i \leq N-1$ under the braid group $B\left(A_{N-1}\right)$ action in Theorem 2.3. Then there is an automorphism $s_{L}$ on $K\left(\mathcal{T}_{N}\right)$ such that

(a) $s_{L(i)}=s_{i}$ for $1 \leq i \leq N-1$.

(b) $s_{\left(s_{L}\left(L^{\prime}\right)\right)}=s_{L} \circ s_{L^{\prime}} \circ s_{L}^{-1}$ if $L^{\prime}$ also satisfies the condition in the theorem.

(c) $s_{\mathscr{D}^{a} L}=s_{L}$ for all $a \in \mathbf{Z}$.

(d) $s_{L}([X])=\left[\left(\mathscr{D}^{a} L\right)^{\circ n} \nabla X\right]$ up to a power of $t$, if $\mathfrak{d}\left(\mathscr{D}^{k} L, X\right)=n \delta(k=a)$ for some $n \geq 0$ and $a \in \mathbf{Z}$.

5. Conjectures. Let $U_{q}^{\prime}(\mathfrak{g})$ be an arbitrary quantum affine algebra. We say that a real simple module $L$ in $\mathscr{C}_{\mathfrak{g}}^{0}$ is a root module if $\mathfrak{d}\left(\mathscr{D}^{k} M, M\right)=$ $\delta(k= \pm 1)$ for any $k$.

Conjecture. For any root module $L \in \mathscr{C}_{\mathfrak{g}}^{0}$, there exists a monoidal autofunctor $\mathscr{S}_{L}$ of $\mathscr{C}_{\mathfrak{g}}^{0}$ which satisfies the following conditions:

(a) $\mathscr{S}_{L}$ satisfies similar properties in Theorem 4.1 and Theorem 4.2 .

(b) (Braid relation) For root modules $L$ and $L^{\prime}$, (1) if $\mathfrak{d}\left(\mathscr{D}^{k} L, L^{\prime}\right)=0$ for any $k \in \mathbf{Z}$, then

$$
\mathscr{S}_{L} \circ \mathscr{S}_{L^{\prime}} \simeq \mathscr{S}_{L^{\prime}} \circ \mathscr{S}_{L}
$$

(2) if $\mathfrak{d}\left(\mathscr{D}^{k} L, L^{\prime}\right)=\delta(k=0)$ for any $k \in \mathbf{Z}$, then

$$
\mathscr{S}_{L} \circ \mathscr{S}_{L^{\prime}} \circ \mathscr{S}_{L} \simeq \mathscr{S}_{L^{\prime}} \circ \mathscr{S}_{L} \circ \mathscr{S}_{L^{\prime}} \text {. }
$$

(c) Let $Q$ be a Q-data, and $L:=\mathcal{F}_{Q}(L(i))$. Then the automorphism of $\mathcal{K}_{t}(\mathfrak{g})$ induced by $\mathscr{S}_{L}$ coincides with $\sigma_{i}$, i.e., the following diagram commutes:

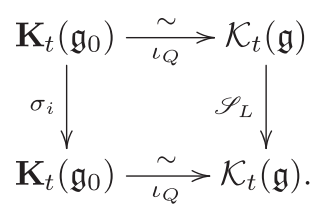

Acknowledgement. The research of Kashiwara was supported by Grant-in-Aid for Scientific Research (B) 15H03608, Japan Society for the Promotion of Science, the research of Kim was supported by the National Research Foundation of Korea(NRF) Grant funded by the Korea government(MSIP) (NRF-2017R1C1B2007824) and the research of $\mathrm{Oh}$ was supported by the Ministry of Education of the Republic of Korea and the NRF of Korea (NRF-2019R1A2C4069647).

\section{References}

[ 1 ] R. Fujita, Affine highest weight categories and quantum affine Schur-Weyl duality of Dynkin quiver types, arXiv:1710.11288.

[ 2 ] R. Fujita, Geometric realization of Dynkin quiver type quantum affine Schur-Weyl duality, Int. Math. Res. Not. IMRN 22 (2020), 8353-8386.

[ 3 ] D. Hernandez, Algebraic approach to $q, t$-characters, Adv. Math. 187 (2004), no. 1, 1-52.

[ 4 ] D. Hernandez and B. Leclerc, Cluster algebras and quantum affine algebras, Duke Math. J. 154 (2010), no. 2, 265-341.

[ 5 ] D. Hernandez and B. Leclerc, Quantum Grothendieck rings and derived Hall algebras, J. Reine Angew. Math. 701 (2015), 77-126.

[ 6 ] S.-J. Kang, M. Kashiwara and M. Kim, Symmetric quiver Hecke algebras and $R$-matrices of quantum affine algebras, II, Duke Math. J. 164 (2015), no. 8, 1549-1602. 
[ 7 ] S.-J. Kang, M. Kashiwara and M. Kim, Symmetric quiver Hecke algebras and R-matrices of quantum affine algebras, Invent. Math. 211 (2018), no. 2, 591-685.

[ 8 ] M. Kashiwara, M. Kim, S.-J. Oh and E. Park, Monoidal categories associated with strata of flag manifolds, Adv. Math. 328 (2018), 9591009.

[ 9 ] M. Kashiwara, M. Kim, S.-J. Oh and E. Park, Cluster algebra structures on module categories over quantum affine algebras, arXiv:1904. 01264.

[ 10 ] M. Kashiwara, M. Kim, S.-J. Oh and E. Park, Simply-laced root systems arising from quantum affine algebras, arXiv:2003.03265.

[11 ] M. Kashiwara and E. Park, Affinizations and Rmatrices for quiver Hecke algebras, J. Eur. Math. Soc. (JEMS) 20 (2018), no. 5, 1161-1193.
[12] S. Kato, Poincaré-Birkhoff-Witt bases and Khovanov-Lauda-Rouquier algebras, Duke Math. J. 163 (2014), no. 3, 619-663.

[13] S. Kato, On the monoidality of Saito reflection functors, Int. Math. Res. Not. IMRN 22 (2020), 8600-8623.

[14] H. Nakajima, Quiver varieties and $t$-analogs of $q$-characters of quantum affine algebras, Ann. of Math. (2) 160 (2004), no. 3, 1057-1097.

[15] Y. Saito, PBW basis of quantized universal enveloping algebras, Publ. Res. Inst. Math. Sci. 30 (1994), no. 2, 209-232.

[ 16 ] M. Varagnolo and E. Vasserot, Perverse sheaves and quantum Grothendieck rings, in Studies in memory of Issai Schur (Chevaleret/Rehovot, 2000), 345-365, Progr. Math., 210, Birkhäuser Boston, Boston, MA, 2003. 